\title{
THE NERNST LAMP IN THE LABORATORY AND SIMPLE EXPERIMENTS ON RADIO-ACTIVITY.*
}

\author{
By F. R. GORTON, \\ Michigan State Normal College, Ypsilanti, Mich.
}

I. The Nernst lamp consists essentially of a specially prepared filament A, Fig. I, called the glower, and a series resistance $\mathrm{B}$, called the ballast. The glower possesses the property of conducting electricity only when hot. Joining a glower and a corresponding ballast across the electric lighting mains and heating the filament in a flame brings it into a conducting condition after which the current quickly carries it to a temperature at which it gives off an intense light. The ballast consists of an iron wire of such a length and size as to offer sufficient resistance while conducting the necessary current. Obviously the glowers and the ballasts must be adapted to the voltage on which they are to operate. These parts of the lamp already described can be had neatly mounted in lamp bodies in which the initial heating of the glower is done automatically by the current. While the complete lamps are excellent for commercial lighting purposes, a more convenient mounting is desirable for use in the laboratory. One form that has been found useful for many purposes is shown in crosssection in Fig. 2. The parts are mounted on part of a porcelain
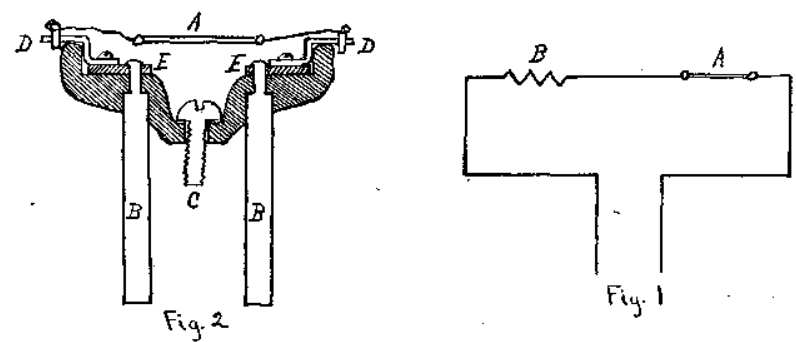

ceiling rosette used in electric wiring. $\mathrm{BB}$ are two brass posts passing through the screw holes in the porcelain and attaching to the small brass plates $\mathrm{EE}$. The joints cannot be soldered on account of the heat produced while the lamp is in operation. To the plates EE are attached the metal extensions DD. These are drilled near the outer ends to receive the small aluminum pins with which each glower is provided. The large screw $C$ serves to connect the lamp to the kind of holder or stand desired. The

*The cuts on ratio-activity are loaned by the Western Journal of Education. 
metal parts can be protected from the intense heat of the glower by filling the holder with plaster of Paris. The electrical connections are made by attaching flexible lamp cord to the ends of the posts $\mathrm{BB}$. These contacts may be soldered. It is advisable to fit pieces of glass tubing over the posts before attaching the wires to prevent short-circuiting. The ballast may be placed anywhere in the circuit. A convenient mount is shown in Fig 3.

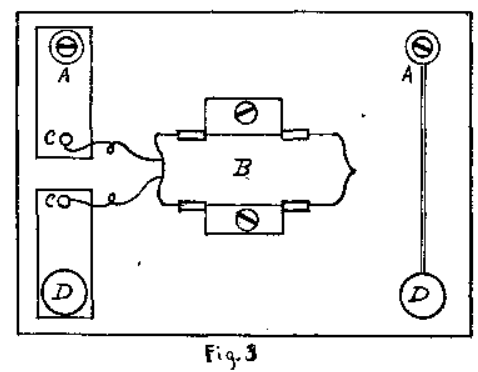

The lamp as described serves admirably as a luminant for projection purposes for pictures five or six feet in diameter. Smaller pictures in a partially darkened room can be distinctly seen by a class. Two IIo-volt glowers serve for projection in a room seating three or four hundred.

Another application can be made in rendering galvanometer deflections visible to a class. Place the lamp about $75 \mathrm{cms}$. in front of a mirror galvanometer and a convex lens whose principal focal length is 50 cms. about $\sigma_{5} \mathrm{cms}$. from the lamp and between the two. A brilliant image of the glower will be reflected upon a screen or scale placed behind the lamp at a distance of about two meters. Of course, other lenses than that mentioned may be used and the correct position of all the parts found by trial. The image is bright enough to be observed without darkening the room.

A brilliant point source for experimental purposes can be produced by placing a slit near, and at right angles to, the glower. For work in optics not requiring a very narrow slit, the glower itself serves very well.

Other uses will suggest themselves to teachers of physics as they come to use this luminant. The glowers and ballasts are made both for direct and alternating currents. They can be procured from the Nernst Lamp Co., of Pittsburg, Pa., or Chicago. The nature of the current and the voltage must be stated in ordering the parts. 
2. Two effects of radio-active substances are easily made use of in the laboratory; viz., the photographic action and the electrical effect due to the radiations emitted by such bodies. Probably the most accessible substance possessing the property in any

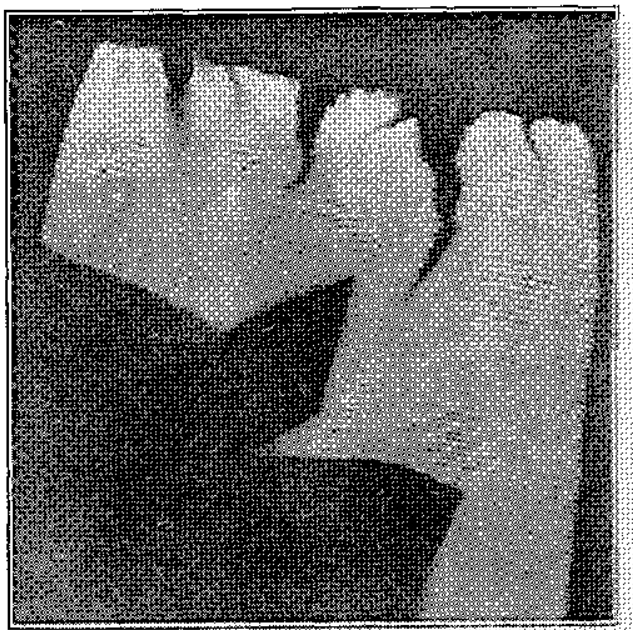

Fig. 4. Effect obtained by placing and ordinary gas mantle upon a photographic plate. The fogging of the plate shows the presence of a radioactive substance which in this case was thorium. Time of expostre, 13 days. Lighter regions produced by interposed pieces of paper.

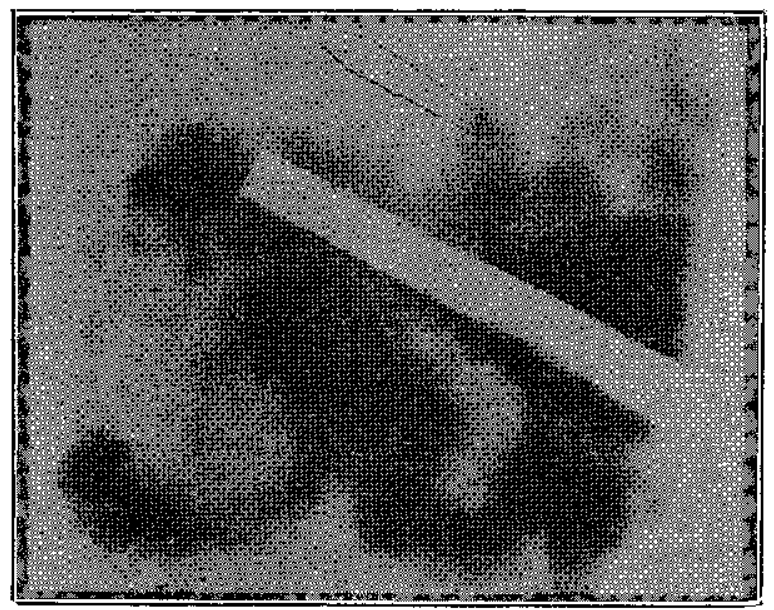

Fig. 5. Plate exposed 13 days to the activity of the ashes of 10 gas mantles, showing presence of radio-active substance and effect of intervening pieces of paper and thin metal strip. 
marked degree is to be found in the Welsbach gas mantle. If such a mantle is laid flat against the film of an ordinary sensitive plate and left in the dark for a week or more before being removed, the process of development will bring out a distinct image of the mantle. See Figs. 4 and 5. The experiment can be made by using the mineral Pitchblende, or Uraninite, which is usually found in mineralogical collections and is kept in stock by dealers in such materials. Good results can be obtained by breaking the rock in small pieces and strewing it around upon the plate. If a coin, for example, is first placed on the plate, a clear spot will result on developing due to the shielding action of the metal. See Fig. 6. The activity of the mantle is due to the presence of

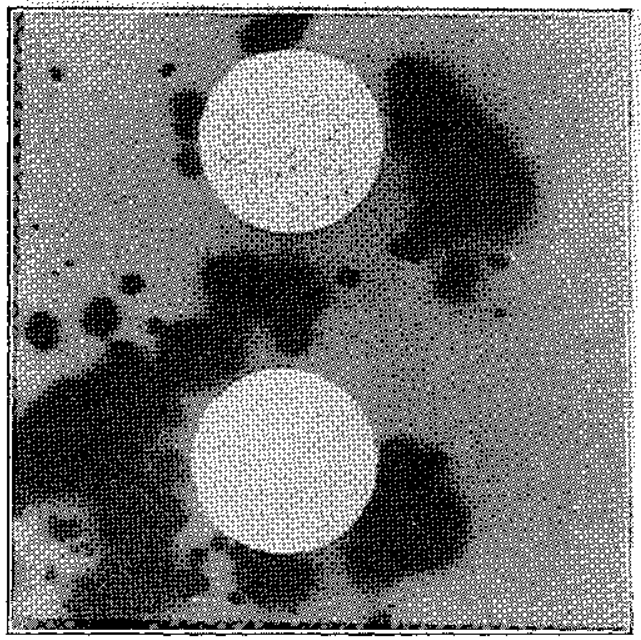

Fig. 6. Restlt obtained by strewing strall pieces of the mineral pitchblende upon a photographic plate. The round spots show where the plate was shielded by the presence of two copper coins.

Thorium, while that of the pitchblende arises from the Uranium contained in it. A part of the activity of the latter may be due, however, to the presence of a trace of Radium which is found in pitchblende. The photographic effect may be produced by radium-cards and radium-pencils sold by dealers in such apparatus at a small price. In order to show the penetrating power of the beta-rays, plates were exposed to the action of a radium card for several days, having intervening sheets of tin-foil and paper. Figure 7 shows comparatively the shielding effect of paper and foil. Figure 8 was made by folding a sheet of tin-foil so as to 
offer two, three, and four thicknesses to the rays.

The electric effect, so-called, is the action of a radio-active substance in rendering the air around it a conductor of electricity. Thus a charge placed on an electroscope will readily escape in the presence of a radio-active substance. In order to detect the influence of a radio-active body on an electroscope, we have only to make the capacity of the instrument small enough to make the loss of charge apparent. While the activity of a gas mantle can be detected, the influence of a piece of pitchblende is greater. In fact, by projecting the leaves of a small gold-leaf electroscope upon a screen, the effect of the mineral can be observed simultaneously by a number of people.

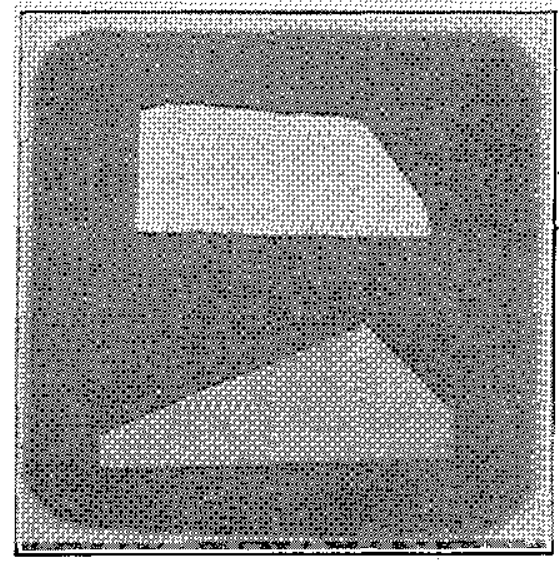

Fig. 7. Effect produced on sensitive photographic plate by a card upon which had been placed a very small quantity of radium. The light regions show the effect of intervening pieces of paper and tin foil. The paper was evidently penetrated by the rays.

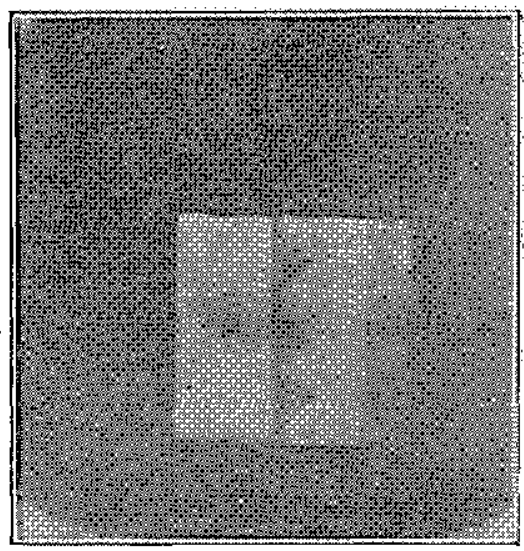

Fig. 8. Effect produced by the card used in obtaining Fig. 7. The light. regions show the penetration of dfferent thicknesses of tin foil. The foil was so folded as to offer two, three, and four layers to the penetrating power of the rays. 